ERHARD CRAmer (Oldenburg)

UDO KAMPS (Oldenburg)

TOMASZ RYCHLIK (Toruń)

\title{
EVALUATIONS OF EXPECTED GENERALIZED ORDER STATISTICS IN VARIOUS SCALE UNITS
}

Abstract. We present sharp upper bounds for the deviations of expected generalized order statistics from the population mean in various scale units generated by central absolute moments. No restrictions are imposed on the parameters of the generalized order statistics model. The results are derived by combining the unimodality property of the uniform generalized order statistics with the Moriguti and Hölder inequalities. They generalize evaluations for specific models of ordered observations.

1. Introduction. The derivation of bounds for moments of order statistics from an iid sample has received a great attention in the literature. Classical results are the Gumbel (1954) and Hartley and David (1954) bounds for the expected sample maximum and the Moriguti (1953) bounds for arbitrary expected order statistics. Some refinements and improvements were proposed, e.g., by Balakrishnan $(1990,1993)$ and Balakrishnan and Bendre (1993). The Moriguti (1953) method, based on the greatest convex minorant, was utilized by Raqab (1997) for $k$ th record values and by Balakrishnan et al. (2001) for progressively type II censored order statistics. Since these results are also based on the Cauchy-Schwarz inequality, the bounds are given in terms of the mean and standard deviation. However, instead of the Cauchy-Schwarz inequality it is near at hand to use its direct generalization, the Hölder inequality with $1 \leq p \leq \infty$. This leads to bounds where the measurement units are powers of absolute central moments of the underlying

2000 Mathematics Subject Classification: Primary 62G30; Secondary 62E15.

Key words and phrases: generalized order statistics, unimodal distribution, Moriguti inequality, Hölder inequality, sharp bound. 
distribution:

$$
\sigma_{p}=\left(E|X-\mu|^{p}\right)^{1 / p} .
$$

In the context of order statistics, Arnold (1985) applied this method to the sample maximum. A generalization to $L$-statistics was proposed by Rychlik (1998). Arnold (1985) and Rychlik (1993) presented analogous evaluations for the maximum and arbitrary $L$-statistics of dependent identically distributed samples, respectively. Recently Raqab and Rychlik (2002) considered $p$-norm bounds for record statistics.

We consider generalized order statistics as a unified approach to models of ordered random variables (cf. Kamps (1995)). In this paper we show that the preceding results can be seen as particular results for generalized order statistics. No restrictions are imposed on the parameters of the generalized order statistics model. The bounds follow from the fundamental observation that densities of uniform generalized order statistics are unimodal (cf. Cramer et al. (2002)), which is thoroughly discussed in Section 2. Section 3 contains the main results of the paper.

2. Preliminaries. Generalized order statistics based on some distribution function $G$ and parameters $\gamma_{1}, \ldots, \gamma_{n}>0$ were introduced by Kamps (1995) via the quantile transformation

$$
X_{r}=G^{-1}\left(U_{r}\right), \quad 1 \leq r \leq n,
$$

where $U_{1}, \ldots, U_{n}$ are generalized order statistics based on the (standard) uniform distribution and the parameters $\gamma_{1}, \ldots, \gamma_{n}$. The joint density function of $U_{1}, \ldots, U_{n}$ is given by

$$
f^{U_{1}, \ldots, U_{n}}\left(u_{1}, \ldots, u_{n}\right)=k\left(\prod_{j=1}^{n-1} \gamma_{j}\right)\left(\prod_{j=1}^{n-1}\left(1-u_{j}\right)^{\gamma_{j}-\gamma_{j+1}-1}\right)\left(1-u_{n}\right)^{\gamma_{n}-1}
$$

on the cone $\left\{\left(u_{1}, \ldots u_{n}\right): 0 \leq u_{1} \leq \ldots \leq u_{n}<1\right\} \subset \mathbb{R}^{n}$ with parameters $n \in \mathbb{N}, \gamma_{1}, \ldots, \gamma_{n}>0$.

It was shown in Cramer and Kamps (2002) that the marginal density of the $r$ th uniform generalized order statistic can be written in terms of a particular Meijer $G$-function, i.e.,

$$
f_{r}(t)=f^{U_{r}}(t)=\left(\prod_{i=1}^{r} \gamma_{i}\right) \mathbf{G}_{r, r}^{r, 0}\left[1-t \mid \begin{array}{c}
\gamma_{1}, \ldots, \gamma_{r} \\
\gamma_{1}-1, \ldots, \gamma_{r}-1
\end{array}\right], \quad t \in[0,1) .
$$

The marginal cumulative distribution function of the $r$ th uniform generalized order statistic is denoted by $F_{r}$. Hence, the cumulative distribution function of $X_{r}=G^{-1}\left(U_{r}\right)$ based on some distribution function $G$ is given by $G_{r}=F_{r} \circ G$. Since only the marginal distribution is considered in the following calculations, and this does not depend on the ordering of the parameters 
$\gamma_{1}, \ldots, \gamma_{r}$, we assume without loss of generality that the parameters are decreasingly ordered, i.e., $\gamma_{1} \geq \ldots \geq \gamma_{r}>0$.

Let $X$ be a random variable with distribution function $G$. Subsequently, we tacitly assume that the expectation $\mu=E X$ and the $p$ th absolute central moment

$$
\sigma_{p}^{p}=E|X-\mu|^{p}=\int_{0}^{1}\left|G^{-1}(u)-\mu\right|^{p} d u, \quad 1 \leq p<\infty,
$$

exist and are finite whenever they are used. Moreover, if the support of $X$ is bounded, we denote the essential supremum of its deviation from the mean by

$$
\sigma_{\infty}=\operatorname{ess} \sup |X-\mu|=\sup \left\{\left|G^{-1}(u)-\mu\right|: u \in(0,1)\right\} .
$$

In the following, the behavior of $f_{r}$ at the endpoints of the interval $(0,1)$ is important. For $r=1$, the density function $f_{1}(t)=\gamma_{1}(1-t)^{\gamma_{1}-1}, t \in(0,1)$, has the limits

$$
\lim _{t \rightarrow 0+} f_{1}(t)=\gamma_{1}>0, \quad \lim _{t \rightarrow 1-} f_{1}(t)= \begin{cases}+\infty & \text { if } \gamma_{1}<1 \\ 1 & \text { if } \gamma_{1}=1 \\ 0 & \text { if } \gamma_{1}>1\end{cases}
$$

If $r \geq 2$, we have

$$
\begin{aligned}
& \lim _{t \rightarrow 0+} f_{r}(t)=0, \\
& \lim _{t \rightarrow 1-} f_{r}(t)= \begin{cases}+\infty & \text { if } \gamma_{r}<1 \text { or } \gamma_{r}=\gamma_{r-1}=1, \\
f_{r}(1) \in(0, \infty) & \text { if } \gamma_{r}=1<\gamma_{r-1}, \\
0 & \text { if } \gamma_{r}>1 .\end{cases}
\end{aligned}
$$

The value of $f_{r}(1)$ in the the case $\gamma_{r}=1<\gamma_{r-1}$ can be calculated explicitly (see Cramer and Kamps (2002)).

Subsequently, we denote the limits of $f_{r}$ at the endpoints of $(0,1)$ by $f_{r}(0)$ and $f_{r}(1)$, respectively. The following theorem is fundamental in deriving bounds on expected generalized order statistics (cf. Cramer et al. (2002)).

Proposition 2.1 The density function of each uniform generalized order statistic is unimodal.

For $r=1$, the density function is strictly increasing, constant and strictly decreasing for $\gamma_{1}<1,=1$, and $>1$, respectively.

For $r \geq 2$ the following result holds: If $\gamma_{r} \leq 1$, then the density function is strictly increasing. Otherwise it is strictly unimodal with a mode in $(0,1)$.

Four cases are distinguished in the following considerations:

(A) $f_{r} \equiv 1$, i.e., $r=1, \gamma_{1}=1$;

(B) $\quad f_{r}$ is strictly decreasing, i.e., $r=1, \gamma_{1}>1$;

(C) $\quad f_{r}$ is strictly increasing with 
(i) $f_{r}(1)<\infty$, i.e., $r \geq 2, \gamma_{r-1}>\gamma_{r}=1$;

(ii) $f_{r}(1)=\infty$, i.e., $r \geq 1, \gamma_{r}<1$ or $r \geq 2, \gamma_{r-1}=\gamma_{r}=1$;

(D) $\quad f_{r}$ is strictly unimodal with a mode $z_{r} \in(0,1)$ and $f_{r}(0)=f_{r}(1)=0$, i.e., $r \geq 2, \gamma_{r}>1$.

3. Bounds via a combination of the Moriguti and Hölder inequalities. We aim now at deriving the projections $P f_{r}$ of the density functions $f_{r}$ onto the family of nondecreasing functions in the space $L^{2}([0,1), d u)$ of square integrable functions on the standard unit interval. Considering the preceding set-ups (A)-(D) and Proposition 2.1, we come up with the following conclusions.

Proposition 3.1 (i) $P f_{r}=1$ in cases (A) and (B);

(ii) $P f_{r}=f_{r}$ in case $(\mathrm{C})$;

(iii) $P f_{r}(\cdot)=f_{r}(\min (\cdot, b))$ in case $(\mathrm{D})$, where $0<b<z_{r}$ is the unique solution of the equation

$$
(1-x) f_{r}(x)=1-F_{r}(x), \quad x \in(0,1) .
$$

Proof. The projection $P f_{r}$ of $f_{r}$ onto the convex cone of nondecreasing functions in $L^{2}([0,1), d u)$ is derived by means of Moriguti's greatest convex minorant method. This is the derivative of the greatest convex minorant of the antiderivative $F_{r}$ of the density function $f_{r}$. A formal justification can be found in Rychlik (2001, pp. 14-16). In cases (A) and (C), $f_{r}$ is actually nondecreasing, and coincides with the projection $P f_{r}$. In case (B), $F_{r}(t)=1-(1-t)^{\gamma_{1}}$ is concave increasing from $F_{r}(0)=0$ to $F_{r}(1)=1$. Therefore its greatest convex minorant is the linear function $\widetilde{F}_{r}(t)=t$, $t \in[0,1]$, with the derivative $\widetilde{F}_{r}^{\prime}=P f_{r} \equiv 1$.

The only nontrivial case is (D), where $F_{r}$ is strictly convex increasing on $\left(0, z_{r}\right)$ and strictly concave increasing on $\left(z_{r}, 1\right)$. The greatest convex minorant $\widetilde{F}_{r}$ of $F_{r}$ is

$$
\widetilde{F}_{r}(t)= \begin{cases}F_{r}(t) & \text { for } t \in[0, b], \\ \frac{1-F_{r}(b)}{1-b}(t-1)+1 & \text { for } t \in[b, 1],\end{cases}
$$

for some $0 \leq b \leq z_{r}$. The case $b=0$ is excluded, because $f_{r}(0)=0$, and $F_{r}$ lies below the line joining $(0,0)$ and $(1,1)$ in a neighborhood of 0 , i.e., $F_{r}(t)<t$ for $t \in(0, \varepsilon)$. Looking for $b$, we consider the linear functions

$$
l_{x}(t)=f_{r}(x)(t-x)+F_{r}(x), \quad t \in \mathbb{R},
$$

tangent to $F_{r}$ at $x \in\left(0, z_{r}\right]$ and evaluate them for $t=1$. Since $l_{x}(1)$ is a 
continuous function in $x$, the values $l_{x}(1)$ strictly increase from $l_{0}(1)=0$ to

$$
\begin{aligned}
l_{z_{r}}(1) & =f_{r}\left(z_{r}\right)\left(1-z_{r}\right)+F_{r}\left(z_{r}\right) \\
& =\int_{0}^{z_{r}} f_{r}(u) d u+\int_{z_{r}}^{1} f_{r}\left(z_{r}\right) d u>\int_{0}^{1} f_{r}(u) d u=F_{r}(1)=1 .
\end{aligned}
$$

Consequently, there exists a unique $b \in\left(0, z_{r}\right)$ such that

$$
l_{b}(1)=F_{r}(b)+(1-b) f_{r}(b)=1
$$

(cf. (1)). Combining this property with (2) we obtain the derivative

$$
P f_{r}(t)=\widetilde{F}_{r}^{\prime}(t)= \begin{cases}f_{r}(t) & \text { for } t \in[0, b], \\ f_{r}(b) & \text { for } t \in[b, 1],\end{cases}
$$

which has the desired representation.

In consequence, we obtain the Moriguti inequality (cf. Rychlik (2001, p. 34))

$$
E X_{r}=\int_{0}^{1} G^{-1}(u) f_{r}(u) d u \leq \int_{0}^{1} G^{-1}(u) P f_{r}(u) d u .
$$

Equality holds in (4) if $G^{-1}$ is constant on each interval where the distribution function $F_{r}$ is greater than its greatest convex minorant. In cases (A) and (C), there is no restriction since $f_{r}=P f_{r}$ so that (4) holds with equality. In case (B), $G^{-1}$ is constant on $(0,1)$, which means that $G$ is concentrated at the single point $\mu$. In the remaining case (D), $G^{-1}$ is constant on the interval $(b, 1)$, implying that $G$ has a jump of probability at least $1-b$ at the right endpoint of its support.

Applying the equalities $\int_{0}^{1} P f_{r}(u) d u=1$ and $\int_{0}^{1}\left[G^{-1}(u)-\mu\right] d u=0$ we find for arbitrary $c \in \mathbb{R}$ the inequality

$$
\begin{aligned}
E X_{r}-\mu & \leq \int_{0}^{1}\left[G^{-1}(u)-\mu\right] P f_{r}(u) d u \\
& =\int_{0}^{1}\left[G^{-1}(u)-\mu\right]\left[P f_{r}(u)-c\right] d u .
\end{aligned}
$$

Choosing $c=1$ in cases (A) and (B), we can evaluate the right hand side of (4) directly:

$$
E X_{r}-\mu \leq \int_{0}^{1}\left[G^{-1}(u)-\mu\right]\left[P f_{r}(u)-1\right] d u=0 .
$$

We have equality in case (A), and the simple relation $E X_{r}=\mu$ holds for every distribution function $G$. In case (B), the bound $E X_{r} \leq \mu$ is attained for 
the one-point distribution with mass at $\mu$. These trivial cases are excluded from the further study.

In the remaining cases (C) and (D), the Hölder inequality is applied to evaluate the upper bound (5). Note that $P f_{r}$ has the common form (3) with $b=1$ under assumption (C). Moreover, $P f_{r}=f_{r}$, and it suffices to evaluate the middle term in (4). The cases $p=1,1<p<\infty$, and $p=\infty$ are considered separately in the subsequent sections.

3.1. Case $p=1$. The relations (5) yield

$$
\begin{aligned}
E X_{r}-\mu & \leq \int_{0}^{1}\left[G^{-1}(u)-\mu\right]\left[P f_{r}(u)-c\right] d u \\
& \leq \sup _{u \in(0,1)}\left|P f_{r}(u)-c\right| \int_{0}^{1}\left|G^{-1}(u)-\mu\right| d u=B_{1}(c) \sigma_{1} .
\end{aligned}
$$

The constant $c$ is chosen so that it minimizes the factor $B_{1}(c)$.

Theorem 3.2. With $b$ defined in (1), we have

$$
\sup _{G} \frac{E X_{r}-\mu}{\sigma_{1}}=B_{1}= \begin{cases}\infty & \text { in case }(\mathrm{C})(\mathrm{ii}), \\ f_{r}(1) / 2<\infty & \text { in case (C)(i), } \\ f_{r}(b) / 2<\infty & \text { in case (D). }\end{cases}
$$

The bounds are not attained, but there are sequences of distributions, e.g., three-point distributions, which attain the bounds in the limit.

Proof. Since $P f_{r}$ is nondecreasing, $P f_{r}(0)=0$, and $P f_{r}(1)=f_{r}(1)<$ $\infty, P f_{r}(1)=\infty$, and $P f_{r}(1)=f_{r}(b)<\infty$ in cases $(\mathrm{C})(\mathrm{i}),(\mathrm{C})(\mathrm{ii})$, and (D), respectively, the upper bounds are immediately determined by calculating

$$
B_{1}=\inf _{c \in \mathbb{R}} B_{1}(c)=\inf _{c \in \mathbb{R}} \sup _{u \in(0,1)}\left|P f_{r}(u)-c\right| .
$$

We show that the bounds are attained.

In case $(\mathrm{C})$, for $0<\varepsilon<1 / 2$, we define a random variable $X$ with the distribution

$$
P\left(X=\mu-\frac{\sigma_{1}}{2 \varepsilon}\right)=P\left(X=\mu+\frac{\sigma_{1}}{2 \varepsilon}\right)=\varepsilon, \quad P(X=\mu)=1-2 \varepsilon .
$$

It is easily checked that $E X=\mu$ and $E|X-\mu|=\sigma_{1}$. Moreover,

$$
\begin{aligned}
\frac{E X_{r}-\mu}{\sigma_{1}} & =\int_{0}^{1} \frac{G^{-1}(u)-\mu}{\sigma_{1}} f_{r}(u) d u=\frac{1}{2 \varepsilon}\left[\int_{1-\varepsilon}^{1} f_{r}(u) d u-\int_{0}^{\varepsilon} f_{r}(u) d u\right] \\
& \geq \frac{f_{r}(1-\varepsilon)-f_{r}(\varepsilon)}{2} \rightarrow \frac{f_{r}(1)}{2}=B_{1} \quad \text { as } \varepsilon \rightarrow 0 .
\end{aligned}
$$


In case (D), for $0<\varepsilon<b$, we choose $X$ so that $P\left(X=\mu-\frac{\sigma_{1}}{2 \varepsilon}\right)=\varepsilon, P(X=\mu)=b-\varepsilon, P\left(X=\mu+\frac{\sigma_{1}}{2(1-b)}\right)=1-b$. This distribution satisfies the moment conditions, and has a jump of height $1-b$ at the right end of its support. Therefore

$$
\begin{aligned}
\frac{E X_{r}-\mu}{\sigma_{1}} & =\int_{0}^{1} \frac{G^{-1}(u)-\mu}{\sigma_{1}} P f_{r}(u) d u=\frac{f_{r}(b)}{2}-\frac{1}{2 \varepsilon} \int_{0}^{\varepsilon} f_{r}(u) d u \\
& \geq \frac{f_{r}(b)-f_{r}(\varepsilon)}{2} \rightarrow \frac{f_{r}(b)}{2}=B_{1} \quad \text { as } \varepsilon \rightarrow 0 .
\end{aligned}
$$

3.2. Case $1<p<\infty$. Applying the Hölder inequality and setting $q=$ $p /(p-1)$ we conclude that

$$
\begin{aligned}
E X_{r}-\mu & \leq \int_{0}^{1}\left[G^{-1}(u)-\mu\right]\left[P f_{r}(u)-c\right] d u \\
& \leq\left\|G^{-1}(u)-\mu\right\|_{p}\left\|P f_{r}-c\right\|_{q} \\
& =\left\|P f_{r}-c\right\|_{q} \sigma_{p}=B_{p}(c) \sigma_{p},
\end{aligned}
$$

where the bounds $B_{p}(c)=\left\|P f_{r}-c\right\|_{q}$ depend on the constants $c \in \mathbb{R}$.

Theorem 3.3. Let $b=1$ in case (C) and let $b$ solve equation (1) in case (D). Then

$$
\sup _{G} \frac{E X_{r}-\mu}{\sigma_{p}}=B_{p}
$$

where

$$
\begin{aligned}
B_{p}^{p}= & \int_{0}^{a}\left[f_{r}(a)-f_{r}(u)\right]^{q} d u+\int_{a}^{b}\left[f_{r}(u)-f_{r}(a)\right]^{q} d u \\
& +(1-b)\left[f_{r}(b)-f_{r}(a)\right]^{q},
\end{aligned}
$$

and $a \in(0, b)$ is the unique solution of the equation

$$
\begin{aligned}
\int_{0}^{a}\left[f_{r}(a)-f_{r}(u)\right]^{q-1} d u= & \int_{a}^{b}\left[f_{r}(u)-f_{r}(a)\right]^{q-1} d u \\
& +(1-b)\left[f_{r}(b)-f_{r}(a)\right]^{q-1} .
\end{aligned}
$$

$A$ distribution function $G$ that attains the bound (8) is given by

$$
G(t)= \begin{cases}0 & \text { if } t \leq \alpha, \\ f_{r}^{-1}\left(f_{r}(a)+\operatorname{sgn}(t-\mu) \frac{B_{p}}{\sigma_{p}}|t-\mu|^{p / q}\right) & \text { if } \alpha \leq t<\beta, \\ 1 & \text { if } t \geq \beta,\end{cases}
$$


for

$$
\alpha=\mu-\sigma_{p}\left[\frac{f_{r}(a)}{B_{p}}\right]^{q / p} \quad \text { and } \quad \beta=\mu+\sigma_{p}\left[\frac{f_{r}(b)-f_{r}(a)}{B_{p}}\right]^{q / p} .
$$

Proof. First we determine the constant $c$ that minimizes the bound in (7). The function $P f_{r}$ is continuous nondecreasing with image $\left[0, f_{r}(b)\right]$. It is obvious that the optimal $c$ fulfils $c \in\left[0, f_{r}(b)\right]$ so that it can be represented as $c=f_{r}(a)$ for a unique $a \in[0, b]$. Define the function

$$
\begin{aligned}
D_{p}(x)= & B_{p}^{p}\left(f_{r}(x)\right)=\left\|P f_{r}-f_{r}(x)\right\|_{q}^{q} \\
= & \int_{0}^{x}\left[f_{r}(x)-f_{r}(u)\right]^{q} d u+\int_{x}^{b}\left[f_{r}(u)-f_{r}(x)\right]^{q} d u \\
& +(1-b)\left[f_{r}(b)-f_{r}(x)\right]^{q}, \quad x \in(0, b) .
\end{aligned}
$$

Its derivative is given by

$$
\begin{aligned}
D_{p}^{\prime}(x)= & q f_{r}^{\prime}(x)\left\{\int_{0}^{x}\left[f_{r}(x)-f_{r}(u)\right]^{q-1} d u\right. \\
& \left.-\int_{x}^{b}\left[f_{r}(u)-f_{r}(x)\right]^{q-1} d u-(1-b)\left[f_{r}(b)-f_{r}(x)\right]^{q-1}\right\} .
\end{aligned}
$$

Both $q$ and $f_{r}^{\prime}(x)$ for $x \in(0, b)$ are positive. Moreover, the first term in braces is equal to 0 at 0 , and it strictly increases in $x$. The sum of the second and third terms is negative, strictly increasing and vanishing at $b$. Therefore there exists a unique $a \in(0, b)$ at which $D_{p}^{\prime}$ changes sign from minus to plus, and thus $D_{p}$ attains its minimum. Equivalently, the point is uniquely determined by equation (9).

Now we show that (10) is the unique distribution function that satisfies both the moment conditions and provides equalities in (7) with the optimal constant. Equalities in the Hölder inequality and in the moment conditions hold if

$$
\frac{G^{-1}(x)-\mu}{\sigma_{p}}=\frac{\left|P f_{r}(x)-f_{r}(a)\right|^{q / p} \operatorname{sgn}\left\{P f_{r}(x)-f_{r}(a)\right\}}{\left\|P f_{r}-f_{r}(a)\right\|_{q}^{q / p}}, \quad x \in(0,1) .
$$

This is a nondecreasing function which defines the distribution function (10). Note that in case (D), it has an atom with mass $1-b$ at the right endpoint of its support, and so the Moriguti inequality is attained as well. In case $(C)$, $P f_{r}=f_{r}$, and the application of the Moriguti inequality is redundant.

In the particular case $p=q=2$, the representation of the bounds simplifies considerably. First of all, from

$$
\left\|P f_{r}-c\right\|_{2}^{2}=\left\|P f_{r}-1\right\|_{2}^{2}+(c-1)^{2}
$$


we deduce that $c=1$ is the optimal constant. This yields

$$
B_{2}^{2}=\left\|P f_{r}-1\right\|_{2}^{2}=\left\|P f_{r}\right\|_{2}^{2}-1=\int_{0}^{b} f_{r}^{2}(u) d u+(1-b) f_{r}^{2}(b)-1 .
$$

The bound is attained by the distribution function

$$
G(t)= \begin{cases}0 & \text { if } t \leq \mu-\frac{\sigma_{2}}{B_{2}}, \\ f_{r}^{-1}\left(1+\frac{B_{2}}{\sigma_{2}}(t-\mu)\right) & \text { if } \mu-\frac{\sigma_{2}}{B_{2}} \leq t<\mu+\frac{\sigma_{2}}{B_{2}}\left[f_{r}(b)-1\right], \\ 1 & \text { if } t \geq \mu+\frac{\sigma_{2}}{B_{2}}\left[f_{r}(b)-1\right] .\end{cases}
$$

3.3. Case $p=\infty$. In this set-up we obtain the upper bound

$$
\begin{aligned}
E X_{r}-\mu & \leq \int_{0}^{1}\left[G^{-1}(u)-\mu\right]\left[P f_{r}(u)-c\right] d u \\
& \leq \sup _{u \in[0,1]}\left|G^{-1}(u)-\mu\right| \int_{0}^{1}\left|P f_{r}(u)-c\right| d u=B_{\infty}(c) \sigma_{\infty}
\end{aligned}
$$

with $B_{\infty}(c)=\left\|P f_{r}-c\right\|_{1}$ depending on the real $c$.

TheOREm 3.4. Let $b=1$ or $b$ be the solution to (1), in cases $(\mathrm{C})$ and (D), respectively. Then

$$
\sup _{G} \frac{E X_{r}-\mu}{\sigma_{\infty}}=B_{\infty}= \begin{cases}f_{r}(b)-1 & \text { if } b \leq 1 / 2, \\ 1-2 F_{r}(1 / 2) & \text { if } b>1 / 2 .\end{cases}
$$

The bound is attained by, e.g., the two-point distribution defined by

$$
\begin{aligned}
P\left(X=\mu-\sigma_{\infty}\right) & =\min \{b, 1 / 2\} \\
& =1-P\left(X=\mu+\sigma_{\infty} \frac{\min \{b, 1 / 2\}}{1-\min \{b, 1 / 2\}}\right) .
\end{aligned}
$$

Proof. First, we derive $c$ by minimizing $B_{\infty}(c)$ in (11). An argument similar to that in the proof of Theorem 3.3 shows that the optimal $c$ can be written as $c=f_{r}(a)$ for some $a \in[0, b]$. In order to determine the optimal $a$, we consider

$$
\begin{aligned}
D_{\infty}(x)= & \int_{0}^{1}\left|P f_{r}(u)-f_{r}(x)\right| d u \\
= & \int_{0}^{x}\left[f_{r}(x)-f_{r}(u)\right] d u+\int_{x}^{b}\left[f_{r}(u)-f_{r}(x)\right] d u \\
& +(1-b)\left[f_{r}(b)-f_{r}(x)\right]
\end{aligned}
$$




$$
\begin{aligned}
& =(2 x-1) f_{r}(x)-2 F_{r}(x)+F_{r}(b)+(1-b) f_{r}(b) \\
& =(2 x-1) f_{r}(x)-2 F_{r}(x)+1 .
\end{aligned}
$$

The last relation follows from (1). Furthermore

$$
D_{\infty}^{\prime}(x)=(2 x-1) f_{r}^{\prime}(x), \quad x \in(0, b) .
$$

Since $f_{r}^{\prime}(x)$ is positive for every $x \in(0, b)$, the only zero of the derivative is $a=1 / 2$ subject to the condition that $1 / 2<b$. Otherwise, the minimum is attained at the right endpoint of the domain, i.e., for $a=b$. Hence, $a=\min \{b, 1 / 2\}$ minimizes (14) and provides the best bound in (11). If $1 / 2 \leq b<1$ (in case $(\mathrm{C})$, in particular), then

$$
B_{\infty}=D_{\infty}(1 / 2)=1-2 F_{r}(1 / 2) .
$$

In case (D) with $b<1 / 2$, we have

$$
B_{\infty}=D_{\infty}(b)=f_{r}(b)-1,
$$

due to (1) again.

We now claim that the bound in (12) is attained by the distribution (13). First we observe that $E X=\mu$ and ess sup $|X-\mu|=\sigma_{\infty}$. Moreover, the distribution function of $X$ has a jump of height at least $1-b$ at the right endpoint, and so it provides equality in the Moriguti inequality (see (5)). If $b \geq 1 / 2$, then

$$
\begin{aligned}
E X_{r}-\mu & =\int_{0}^{1}\left[G^{-1}(u)-\mu\right]\left[P f_{r}(u)-f_{r}(1 / 2)\right] d u \\
& =\sigma_{\infty} \int_{0}^{1}\left|P f_{r}(u)-f_{r}(1 / 2)\right| d u=D_{\infty}(1 / 2) \sigma_{\infty}=B_{\infty} \sigma_{\infty} .
\end{aligned}
$$

Otherwise

$$
\begin{aligned}
E X_{r}-\mu & =\int_{0}^{b}\left[G^{-1}(u)-\mu\right]\left[P f_{r}(u)-f_{r}(b)\right] d u \\
& =\sigma_{\infty} \int_{0}^{1}\left|P f_{r}(u)-f_{r}(b)\right| d u=D_{\infty}(b) \sigma_{\infty}=B_{\infty} \sigma_{\infty} .
\end{aligned}
$$

This ends the proof.

Acknowledgements. The authors are grateful to the Deutsche Forschungsgemeinschaft (DFG) for supporting their joint work. The third author was also partially supported by the KBN (Polish State Committee for Scientific Research) Grant No. 5 P03A 01220. 


\section{References}

B. C. Arnold (1985), p-Norm bounds on the expectation of the maximum of possibly dependent sample, J. Multivariate Anal. 17, 316-332.

N. Balakrishnan (1990), Improving the Hartley-David-Gumbel bound for the mean of extreme order statistics, Statist. Probab. Lett. 9, 291-294.

N. Balakrishnan (1993), A simple application of binomial-negative binomial relationship in the derivation of sharp bounds for moments of order statistics based on greatest convex minorants, ibid. 18, 301-305.

N. Balakrishnan and S. M. Bendre (1993), Improved bounds for expectations of linear functions of order statistics, Statistics 24, 161-165.

N. Balakrishnan, E. Cramer and U. Kamps (2001), Bounds for means and variances of progressive type II censored order statistics, Statist. Probab. Lett. 54, 301-315.

E. Cramer and U. Kamps (2002), Marginal distributions of sequential and generalized order statistics, submitted.

E. Cramer, U. Kamps and T. Rychlik (2002), Unimodality of uniform generalized order statistics, with applications to mean bounds, submitted.

E. J. Gumbel (1954), The maxima of the mean largest value and of the range, Ann. Math. Statist. 25, 76-84.

H. O. Hartley and H. A. David (1954), Universal bounds for mean range and extreme observation, Ann. Math. Statist. 25, 85-99.

U. Kamps (1995), A Concept of Generalized Order Statistics, Teubner, Stuttgart.

S. Moriguti (1953), A modification of Schwarz's inequality, with applications to distributions, Ann. Math. Statist. 24, 107-113.

M. Z. Raqab (1997), Bounds based on greatest convex minorants for moments of record values, Statist. Probab. Lett. 36, 35-41.

M. Z. Raqab and T. Rychlik (2002), Sharp bounds for the mean of the kth record value, Comm. Statist. Theory Methods, to appear.

T. Rychlik (1993), Sharp bounds for expectations of L-estimates from dependent samples, ibid. 22, 1053-1068; Correction: ibid. 23, 305-306.

T. Rychlik (1998), Bounds for expectations of L-estimates, in: Order Statistics: Theory \& Methods, N. Balakrishnan and C. R. Rao (eds.), Handbook of Statistics Vol. 16, North-Holland, Amsterdam, 105-145.

T. Rychlik (2001), Projecting Statistical Functionals, Lecture Notes in Statist. 160, Springer, New York.

Department of Mathematics

University of Oldenburg

26111 Oldenburg, Germany

E-mail: cramer@mathematik.uni-oldenburg.de kamps@mathematik.uni-oldenburg.de
Institute of Mathematics Polish Academy of Sciences Chopina 12

87-100 Toruń, Poland E-mail: trychlik@impan.gov.pl

Received on 25.4.2002; 